Research Article

\title{
Fixed-Time Synchronization of Neural Networks with Discrete Delay
}

\author{
Shuai Liu, ${ }^{1}$ Chuan Chen $\mathbb{D}^{2,3}$ and Haipeng Peng $\mathbb{D}^{4}$ \\ ${ }^{1}$ School of Electronic Engieering, Beijing University of Posts and Telecommunications, Beijing 100876, China \\ ${ }^{2}$ School of Cyber Security, Qilu University of Technology (Shandong Academy of Sciences), Jinan 250353, China \\ ${ }^{3}$ Shandong Provincial Key Laboratory of Computer Networks, \\ Shandong Computer Science Center (National Supercomputer Center in Jinan), \\ Qilu University of Technology (Shandong Academy of Sciences), Jinan 250014, China \\ ${ }^{4}$ Information Security Center, State Key Laboratory of Networking and Switching Technology, \\ Beijing University of Posts and Telecommunications, Beijing 100876, China \\ Correspondence should be addressed to Chuan Chen; chenchuan.3000@163.com
}

Received 22 May 2020; Revised 14 July 2020; Accepted 27 July 2020; Published 20 August 2020

Academic Editor: Mariano Torrisi

Copyright (c) 2020 Shuai Liu et al. This is an open access article distributed under the Creative Commons Attribution License, which permits unrestricted use, distribution, and reproduction in any medium, provided the original work is properly cited.

In this paper, we establish a new fixed-time stability theorem, which provides a novel fixed-time stability criterion and a novel upper bound estimate formula for the settling time. Numerical simulations show that the upper bound estimate for the settling time in this paper is tighter than those given in the existing fixed-time stability theorems. By designing a simple feedback controller, the fixed-time synchronization of neural networks with discrete delay is investigated based on the fixed-time stability theorem established in this paper. A numerical example is included to validate the effectiveness of the obtained theoretical results.

\section{Introduction}

Neural networks can be described by differential equations [1], and they usually present complex dynamic behaviors [2]. The stability and synchronization of neural networks can be applied in many fields, such as associative memory [3], secure communication [4], and image encryption [5]. In recent years, considerable results have been reported about the stability and synchronization of neural networks [6-12].

Different from infinite-time synchronization (including asymptotic synchronization and exponential synchronization), finite-time synchronization [13] requires that the trajectories of the error system tend to the origin after some finite time, which is called the settling time. Considering the control cost, finite-time synchronization is more feasible and valuable than infinite-time synchronization in the engineering fields. Recently, the finite-time synchronization of neural networks has been intensively studied [14-22]. In [14], the finite-time synchronization of neural networks with mixed delays and perturbations was investigated. In [19], the finite-time synchronization of Cohen-Grossberg neural networks was studied via delayed feedback control.

The drawback of finite-time synchronization is that the settling time varies with the initial conditions of the driveresponse systems. Since different initial conditions lead to different settling times, the corresponding settling times will be confusing when we consider many different initial conditions. Moreover, if the initial conditions of the studied dynamical systems are unknown beforehand, it is even impossible to estimate the settling times. Therefore, it will be desirable that finite-time synchronization can be achieved in a fixed time interval, irrespective of the initial conditions of the studied systems.

In 2012, a new concept named "fixed-time stability" was introduced by Polyakov [23]. Fixed-time stability is a kind of special finite-time stability actually, and its settling time is bounded by a fixed constant for any initial conditions of the studied systems. As we know, the synchronization problem of the drive-response systems can be converted into the stability problem of the error system, so the related fixed-time stability 
theorems $[23,24]$ can be used to investigate the fixed-time synchronization of nonlinear systems [25-28]. The fixed-time synchronization of nonlinear systems has important applications in secure communication and image encryption. Take secure communication for example: when can the ciphertext signals be decrypted correctly without considering the specific initial values of the drive-response systems? The solution to this problem must be attributed to the fixed-time synchronization control of the drive-response systems. That is to say, for the drive-response systems, the upper bound estimate for the settling time of the fixed-time synchronization can be used to adjust the start time of correct decryption.

As typical nonlinear systems, neural networks usually present some strange dynamic behaviors, so the fixed-time synchronization of neural networks can also be applied in many fields, such as secure communication and image encryption. Based on the fixed-time stability theorems in $[23,24,29]$, there have been some publications about the fixed-time synchronization of neural networks [29-34]. It should be pointed out that the upper bound estimate formulas for the settling time given in $[23,24,29]$ are very inaccurate. Owing to the limitations of theoretical analysis, it is difficult to obtain the least upper bound estimate for the settling time. However, from an application point of view, it is important to derive a tighter upper bound estimate for the settling time.

Inspired by the aforementioned discussion, in this paper, we establish a new fixed-time stability theorem, which provides a novel fixed-time stability criterion and a novel upper bound estimate formula for the settling time. Numerical simulations show that the upper bound estimate for the settling time in this paper is tighter than those given in $[23,24,29]$. By utilizing the established fixed-time stability theorem, the fixed-time synchronization of neural networks with discrete delay is studied. A numerical example is included to show the effectiveness of the obtained theoretical results.

\section{Preliminaries}

Consider the following neural network with discrete delay:

$$
\dot{\xi}_{i}(t)=-\varrho_{i} \xi_{i}(t)+\sum_{j=1}^{n} a_{i j} f_{j}\left(\xi_{j}(t)\right)+\sum_{j=1}^{n} b_{i j} f_{j}\left(\xi_{j}(t-\tau(t))\right)+I_{i},
$$

$i=1,2, \cdots, n$, where $\xi_{i}(t)$ expresses the state of neuron; $\varrho_{i}>0$ denotes the rate of neuron self-inhibition; $a_{i j}$ and $b_{i j}$ denote connection weights; $f_{j}(\cdot)$ represents the activation function; discrete delay $\tau(t)$ satisfies $0 \leq \tau(t) \leq \tau$, where $\tau$ is a positive constant; and $I_{i}$ stands for the external input. The initial condition of system (1) is assumed to be $\xi(\nu)=\varphi(\nu)$ $\in C\left([-\tau, 0], R^{n}\right)$, where $\xi(\nu)=\left(\xi_{1}(\nu), \xi_{2}(\nu), \ldots, \xi_{n}(\nu)\right)^{T}$.

In this paper, system (1) is the drive system, and the corresponding response system is

$$
\begin{aligned}
\dot{\eta}_{i}(t)= & -\varrho_{i} \eta_{i}(t)+\sum_{j=1}^{n} a_{i j} f_{j}\left(\eta_{j}(t)\right) \\
& +\sum_{j=1}^{n} b_{i j} f_{j}\left(\eta_{j}(t-\tau(t))\right)+I_{i}+u_{i}(t),
\end{aligned}
$$

$i=1,2, \cdots, n$, where $u_{i}(t)$ stands for the controller. Let $\eta(t)=\left(\left(\eta_{1}(t), \eta_{2}(t), \cdots, \eta_{n}(t)\right)^{T}\right.$; the initial condition of MNN (2) is $\eta(\nu)=\phi(\nu) \in C\left([-\tau, 0], R^{n}\right)$.

The synchronization errors between systems (1) and (2) are defined by $e_{i}(t)=\eta_{i}(t)-\xi_{i}(t), i=1,2, \cdots, n$; then, the error system should be

$$
\dot{e}_{i}(t)=-\varrho_{i} e_{i}(t)+F_{i}(t)+u_{i}(t)
$$

$i=1,2, \cdots, n$, where

$$
\begin{aligned}
F_{i}(t)= & \sum_{j=1}^{n} a_{i j} f_{j}\left(\eta_{j}(t)\right)-\sum_{j=1}^{n} a_{i j} f_{j}\left(\xi_{j}(t)\right) \\
& +\sum_{j=1}^{n} b_{i j} f_{j}\left(\eta_{j}(t-\tau(t))\right)-\sum_{j=1}^{n} b_{i j} f_{j}\left(\xi_{j}(t-\tau(t))\right) .
\end{aligned}
$$

Let $e(t)=\left(e_{1}(t), e_{2}(t), \cdots, e_{n}(t)\right)^{T}$; then, the initial condition of system (3) should be $e(\nu)=\phi(\nu)-\varphi(\nu)$, $\nu \in[-\tau, 0]$.

Throughout this paper, the following assumptions will be needed:

A1. For $\forall \mu, \nu \in R$, there exist constants $l_{j}>0$ such that

$$
\left|f_{j}(\mu)-f_{j}(\nu)\right| \leq l_{j}|\mu-\nu|, \quad j=1,2, \cdots, n .
$$

A2. For $\forall j \in\{1,2, \cdots, n\}$, activation function $f_{j}(\cdot)$ satisfies $\left|f_{j}(\cdot)\right| \leq M_{j}$, where $M_{j}>0$ is a constant.

Lemma 1. $\left|F_{i}(t)\right| \leq \sum_{j=1}^{n}\left|a_{i j}\right| \cdot l_{j}\left|e_{j}(t)\right|+2 \sum_{j=1}^{n}\left|b_{i j}\right| M_{j}$, $i=1,2, \cdots, n$.

Proof. Based on assumptions $\mathbf{A 1}$ and $\mathbf{A 2}$, the proof is obvious.

Lemma 2 (see [35]). If $y_{1}, y_{2}, \cdots, y_{n} \geq 0,0<\rho \leq 1, \sigma>1$, then we have

$$
\begin{aligned}
& \sum_{i=1}^{n} y_{i}^{\rho} \geq\left(\sum_{i=1}^{n} y_{i}\right)^{\rho}, \\
& \sum_{i=1}^{n} y_{i}^{\sigma} \geq n^{1-\sigma}\left(\sum_{i=1}^{n} y_{i}\right)^{\sigma} .
\end{aligned}
$$

Definition 1. The origin of system (3) can achieve finitetime stability, if there is a constant $T(e(0))>0$ such that $\lim _{t \longrightarrow T}(e(0))\|e(t)\|=0$ and $\|e(t)\| \equiv 0$ for $\forall t>T(e(0))$, where $T(e(0))$ is called the settling time.

Definition 2. The origin of system (3) can achieve fixed-time stability, if two conditions can be satisfied: (a) the origin of system (3) can achieve finite-time stability; (b) for any $e(0)$, there is a fixed constant $T_{\max }>0$ such that $T(e(0)) \leq T_{\max }$. 
Lemma 3 (see [23]). Suppose that $V(\cdot): R^{n} \longrightarrow R_{+} \cup\{0\}$ is a continuous radically unbounded function and satisfies the following:

(1) $V(z)=0 \Longleftrightarrow z=0$

(2) $\dot{V}(e(t)) \leq-\alpha V^{\rho}(e(t))-\beta V^{\sigma}(e(t))$ for any $e(t)$, where $\alpha, \beta>0,0<\rho<1$ and $\sigma>1$

Then, the origin of system (3) can achieve fixed-time stability, and

$$
T_{\max }^{1}=\frac{1}{\alpha(1-\rho)}+\frac{1}{\beta(\sigma-1)} .
$$

Lemma 4 (see [29]). Suppose that $V(\cdot): R^{n} \longrightarrow R_{+} \cup\{0\}$ is a continuous radically unbounded function and satisfies the following:

(1) $V(z)=0 \Longleftrightarrow z=0$

(2) $\dot{V}(e(t)) \leq-\alpha V^{\rho}(e(t))-\beta V^{\sigma}(e(t))$ for any $e(t)$, where $\alpha, \beta>0,0<\rho<1$ and $\sigma>1$

Then, the origin of system (3) can achieve fixed-time stability, and

$$
T_{\max }^{2}=\frac{1}{\alpha} \cdot\left(\frac{\alpha}{\beta}\right)^{(1-\rho) /(\sigma-\rho)}\left(\frac{1}{1-\rho}+\frac{1}{\sigma-1}\right) .
$$

Lemma 5 (see [24]). Suppose that $V(\cdot): R^{n} \longrightarrow R_{+} \cup\{0\}$ is a continuous radically unbounded function and satisfies the following

(1) $V(z)=0 \Longleftrightarrow z=0$

(2) $\dot{V}(e(t)) \leq-\alpha V^{\rho}(e(t))-\beta V^{\sigma}(e(t))$ for any $e(t)$, where $\alpha, \beta>0, \rho=1-(1 / 2 \delta)$ and $\sigma=1+(1 / 2 \delta)$ $(\delta>1)$

Then, the origin of system (3) can achieve fixed-time stability, and

$$
T_{\max }^{3}=\frac{\pi \delta}{\sqrt{\alpha \beta}}
$$

Lemma 6 (see [36]). Suppose $V(\cdot): R^{n} \longrightarrow R_{+} \cup\{0\}$ is C-regular. If $V(\cdot)$ satisfies

$$
\dot{V}(e(t)) \leq-\alpha V^{\rho}(e(t)),
$$

for any $e(t)$, where $\alpha>0$ and $0<\rho<1$ are two constants, then the origin of system (3) can achieve finite-time stability, and the settling time $T(e(0))$ is given by

$$
T(e(0))=\frac{V^{1-\rho}(e(0))}{\alpha(1-\rho)} .
$$

\section{Main Results}

First, we derive a new fixed-time stability theorem, which provides a novel fixed-time stability criterion and a novel upper bound estimate formula for the settling time.
Theorem 1. Suppose that $V(\cdot): R^{n} \longrightarrow R_{+} \cup\{0\}$ is a continuous radically unbounded function and satisfies the following:

(1) $V(z)=0 \Longleftrightarrow z=0$

(2) $\dot{V}(e(t)) \leq-\alpha V^{\rho}(e(t))-\beta V^{\sigma}(e(t))-\gamma V(e(t))$ for any $e(t)$, where $\alpha, \beta, \gamma>0,0<\rho<1$ and $\sigma>1$

Then, the origin of system (3) can achieve fixed-time stability, and

$$
T_{\max }^{4}=\frac{1}{\gamma(1-\rho)} \ln \left(1+\frac{\gamma}{\alpha}\right)+\frac{1}{\beta(\sigma-1)} .
$$

Proof. It is obvious that

$$
\begin{aligned}
\dot{V}(e(t)) & \leq-\alpha V^{\rho}(e(t))-\beta V^{\sigma}(e(t))-\gamma V(e(t)) \\
& \leq-\alpha V^{\rho}(e(t))-\beta V^{\sigma}(e(t)) .
\end{aligned}
$$

Based on Lemma 3, the origin of system (3) can achieve fixed-time stability.

Let $W(s)=V^{1-\rho}(s)$; then, we have

$$
\dot{V}(s)=\frac{1}{1-\rho} \dot{W}(s) V^{\rho}(s) .
$$

Therefore,

$$
\begin{aligned}
& \frac{1}{1-\rho} \dot{W}(e(t)) V^{\rho}(e(t)) \\
& \quad \leq-\alpha V^{\rho}(e(t))-\beta V^{\sigma}(e(t))-\gamma V(e(t)) .
\end{aligned}
$$

Since $V(s)=W^{1 /(1-\rho)}(s)$, it follows that

$$
\begin{aligned}
\dot{W}(e(t)) & \leq(1-\rho)\left[-\alpha-\beta V^{\sigma-\rho}(e(t))-\gamma V^{1-\rho}(e(t))\right] \\
& =(1-\rho)\left[-\alpha-\beta W^{(\sigma-\rho) /(1-\rho)}(e(t))-\gamma W(e(t))\right] .
\end{aligned}
$$

This means

$\frac{\mathrm{d} t}{\mathrm{~d} W(e(t))} \geq \frac{1}{(1-\rho)\left[-\alpha-\beta W^{(\sigma-\rho) /(1-\rho)}(e(t))-\gamma W(e(t))\right]}$.

So we can obtain that

$$
\begin{aligned}
T(e(0)) & \leq \int_{W(e(0))}^{0} \frac{1}{(1-\rho)\left(-\alpha-\beta w^{(\sigma-\rho) /(1-\rho)}-\gamma w\right)} \mathrm{d} w \\
& =\frac{1}{1-\rho} \int_{0}^{W(e(0))} \frac{1}{\alpha+\beta w^{(\sigma-\rho) /(1-\rho)}+\gamma w} \mathrm{~d} w .
\end{aligned}
$$

Two cases will be considered separately:

(1) If $0 \leq V(e(0)) \leq 1$, we have $0 \leq W(e(0))=V^{1-\rho}$ $(e(0)) \leq 1$. Then, 


$$
\begin{aligned}
T(e(0)) & \leq \frac{1}{1-\rho} \int_{0}^{W(e(0))} \frac{1}{\alpha+\gamma w} \mathrm{~d} w \\
& =\frac{1}{\gamma(1-\rho)} \ln \left(1+\frac{\gamma}{\alpha} W(e(0))\right) \leq \frac{1}{\gamma(1-\rho)} \ln \left(1+\frac{\gamma}{\alpha}\right) .
\end{aligned}
$$$$
\text { (2) If } V(e(0))>1 \text {, we have } W(e(0))=V^{1-\rho}(e(0))>1 \text {. }
$$

$$
\begin{aligned}
T(e(0)) & \leq \frac{1}{1-\rho}\left[\int_{0}^{1} \frac{1}{\alpha+\beta w^{(\sigma-\rho) /(1-\rho)}+\gamma w} \mathrm{~d} w+\int_{1}^{W(e(0))} \frac{1}{\alpha+\beta w^{(\sigma-\rho) /(1-\rho)}+\gamma w} \mathrm{~d} w\right] \\
& \leq \frac{1}{1-\rho}\left[\int_{0}^{1} \frac{1}{\alpha+\gamma w} \mathrm{~d} w+\int_{1}^{W(e(0))} \frac{1}{\beta w^{(\sigma-\rho) /(1-\rho)}} \mathrm{d} w\right] \\
& =\frac{1}{\gamma(1-\rho)} \ln \left(1+\frac{\gamma}{\alpha}\right)+\frac{1}{\beta(1-\rho)}\left[\frac{1-\rho}{\sigma-1}-\frac{(1-\rho) W^{(1-\sigma) /(1-\rho)}(x(0))}{\sigma-1}\right] \\
& \leq \frac{1}{\gamma(1-\rho)} \ln \left(1+\frac{\gamma}{\alpha}\right)+\frac{1}{\beta(\sigma-1)} .
\end{aligned}
$$

Then, we have

$$
T_{\max }^{4}=\frac{2}{\gamma(1-\rho)} \ln \left(1+\frac{\gamma}{\alpha}\right)+\frac{2}{\beta(\sigma-1)} .
$$

Remark 1. Since $\ln (1+(\gamma / \alpha))<(\gamma / \alpha)$, we have $(1 /(\gamma(1-$ $\rho))) \ln (1+(\gamma / \alpha))+(1 /(\beta(\sigma-1)))<(1 /(\alpha(1-\rho)))+(1 /$ $(\beta(\sigma-1)))$. Therefore, compared with Lemma 3, Theorem 1 can give $T_{\max }$ a more accurate estimate. Moreover, if $\alpha \ll \gamma$, $\ln (1+(\gamma / \alpha)) \ll(\gamma / \alpha)$.

Next, we investigate the fixed-time synchronization of systems (1) and (2) by means of the following controller:

$$
\begin{aligned}
u_{i}(t)= & -k_{i} e_{i}(t)-q_{i} \operatorname{sign}\left(e_{i}(t)\right)-r_{i} \operatorname{sign}\left(e_{i}(t)\right)\left|e_{i}(t)\right|^{\xi} \\
& -s_{i} \operatorname{sign}\left(e_{i}(t)\right)\left|e_{i}(t)\right|^{\eta},
\end{aligned}
$$

$i=1,2, \cdots, n$, where $k_{i}$ and $q_{i}$ will be determined later, $r_{i}$ and $s_{i}$ are arbitrary positive constants, $0<\xi<1$, and $\eta>1$.
Theorem 2. Suppose assumptions A1 and A2 hold. If control gains $k_{i}$ and $q_{i}$ satisfy $k_{i}>\sum_{j=1}^{n}\left(\left(\left|a_{i j}\right| l_{j}+\left|a_{j i}\right| l_{i}\right) / 2\right)-\varrho_{i}$, $q_{i} \geq 2 \sum_{j=1}^{n}\left|b_{i j}\right| M_{j}, \quad i=1,2, \cdots, n$, systems (1) and (2) can realize fixed-time synchronization under controller (22). In addition,

$$
T_{\max }^{4}=\frac{2}{\gamma(1-\xi)} \ln \left(1+\frac{\gamma}{\alpha}\right)+\frac{2}{\beta(\eta-1)},
$$

where $\alpha=\min _{i}\left\{r_{i}\right\} 2^{(\xi+1) / 2}, \beta=\min _{i}\left\{s_{i}\right\} n^{(1-\eta) / 2} \cdot 2^{(\eta+1) / 2}$, and $\gamma=\min _{i}\left\{2 \varrho_{i}+2 k_{i}-\sum_{j=1}^{n}\left(\left|a_{i j}\right| l_{j}+\left|a_{j i}\right| l_{i}\right)\right\}$.

Proof. We choose the following Lyapunov function:

$$
V(e(t))=\frac{1}{2} e^{T}(t) e(t)=\frac{1}{2} \sum_{i=1}^{n}\left(e_{i}(t)\right)^{2} .
$$

The derivative of $V(e(t))$ is

$$
\begin{aligned}
\dot{V}(e(t))= & \sum_{i=1}^{n} e_{i}(t) e_{i}(t) \\
= & \sum_{i=1}^{n} e_{i}(t) \cdot\left[-\varrho_{i} e_{i}(t)+F_{i}(t)+u_{i}(t)\right] \\
\leq & -\sum_{i=1}^{n} \varrho_{i}\left(e_{i}(t)\right)^{2}+\sum_{i=1}^{n}\left|e_{i}(t)\right|\left[\sum_{j=1}^{n}\left|a_{i j}\right| \cdot l_{j}\left|e_{j}(t)\right|+2 \sum_{j=1}^{n}\left|b_{i j}\right| M_{j}\right] \\
& -\sum_{i=1}^{n} k_{i}\left(e_{i}(t)\right)^{2}-\sum_{i=1}^{n} q_{i}\left|e_{i}(t)\right|-\sum_{i=1}^{n} r_{i}\left|e_{i}(t)\right|^{\xi+1}-\sum_{i=1}^{n} s_{i}\left|e_{i}(t)\right|^{\eta+1}
\end{aligned}
$$




$$
\begin{aligned}
\leq & -\sum_{i=1}^{n}\left(\varrho_{i}+k_{i}-\sum_{j=1}^{n} \frac{\left|a_{i j}\right| l_{j}+\left|a_{j i}\right| l_{i}}{2}\right)\left(e_{i}(t)\right)^{2}+\sum_{i=1}^{n}\left(2 \sum_{j=1}^{n}\left|b_{i j}\right| M_{j}-q_{i}\right)\left|e_{i}(t)\right| \\
& -\sum_{i=1}^{n} r_{i}\left|e_{i}(t)\right|^{\xi+1}-\sum_{i=1}^{n} s_{i}\left|e_{i}(t)\right|^{\eta+1},
\end{aligned}
$$

where Lemma 1 has been used.

$$
\begin{aligned}
\dot{V}(e(t)) \leq & -\min _{i}\left\{r_{i}\right\} \sum_{i=1}^{n}\left(\left|e_{i}(t)\right|^{2}\right)^{(\xi+1) / 2}-\min _{i}\left\{s_{i}\right\} \sum_{i=1}^{n}\left(\left|e_{i}(t)\right|^{2}\right)^{(\eta+1) / 2} \\
& -\min _{i}\left\{\varrho_{i}+k_{i}-\sum_{j=1}^{n} \frac{\left|a_{i j}\right| l_{j}+\left|a_{j i}\right| l_{i}}{2}\right\} \sum_{i=1}^{n}\left(e_{i}(t)\right)^{2} \\
\leq & -\min _{i}\left\{r_{i}\right\}\left(\sum_{i=1}^{n}\left|e_{i}(t)\right|^{2}\right)^{(\xi+1) / 2}-\min _{i}\left\{s_{i}\right\} n^{(1-\eta) / 2} \sum_{i=1}^{n}\left(\left|e_{i}(t)\right|^{2}\right)^{(\eta+1) / 2} \\
& -\min _{i}\left\{\varrho_{i}+k_{i}-\sum_{j=1}^{n} \frac{\left|a_{i j}\right| l_{j}+\left|a_{j i}\right| l_{i}}{2}\right\} \sum_{i=1}^{n}\left(e_{i}(t)\right)^{2} \\
= & -\min _{i}\left\{r_{i}\right\} 2^{(\xi+1) / 2} V^{(\xi+1) / 2}(e(t))-\min _{i}\left\{s_{i}\right\} n^{(1-\eta) / 2} \cdot 2^{(\eta+1) / 2} V^{(\eta+1) / 2}(e(t)) \\
& -2 \min _{i}\left\{\varrho_{i}+k_{i}-\sum_{j=1}^{n} \frac{\left|a_{i j}\right| l_{j}+\left|a_{j i}\right| l_{i}}{2}\right\} V(e(t)),
\end{aligned}
$$

where Lemma 2 has been used.

Let $\alpha=\min _{i}\left\{r_{i}\right\} 2^{(\xi+1) / 2}, \quad \beta=\min _{i}\left\{s_{i}\right\} n^{(1-\eta) / 2} \cdot 2^{(\eta+1) / 2}$, and $\gamma=\min _{i}\left\{2 \varrho_{i}+2 k_{i}-\sum_{j=1}^{n}\left(\left|a_{i j}\right| l_{j}+\left|a_{j i}\right| l_{i}\right)\right\}$. Then, we have

$$
\dot{V}(e(t)) \leq-\alpha V^{(\xi+1) / 2}(e(t))-\beta V^{(\eta+1) / 2}(e(t))-\gamma V(e(t)) .
$$

It is obvious that $0<((\xi+1) / 2)<1$ and $((\eta+1) / 2)>1$. Since $k_{i}>\sum_{j=1}^{n}\left(\left(\left|a_{i j}\right| l_{j}+\left|a_{j i}\right| l_{i}\right) / 2\right)-\varrho_{i}$, we have $\gamma>0$. Based on Theorem 1, the origin of system (3) can achieve fixedtime stability. In addition,

$$
\begin{aligned}
T_{\max }^{4} & =\frac{1}{\gamma(1-((\xi+1) / 2))} \ln \left(1+\frac{\gamma}{\alpha}\right)+\frac{1}{\beta(((\eta+1) / 2)-1)} \\
& =\frac{2}{\gamma(1-\xi)} \ln \left(1+\frac{\gamma}{\alpha}\right)+\frac{2}{\beta(\eta-1)} .
\end{aligned}
$$

Corollary 1. Suppose assumptions $\boldsymbol{A 1}$ and $\boldsymbol{A 2}$ hold. If control gains $k_{i}$ and $q_{i}$ satisfy $k_{i}>\sum_{j=1}^{n}\left(\left(\left|a_{i j}\right| l_{j}+\left|a_{j i}\right| l_{i}\right) /\right.$ 2) $-\varrho_{i}, q_{i} \geq 2 \sum_{j=1}^{n}\left|b_{i j}\right| M_{j}, i=1,2, \cdots, n$, systems (1) and (2) can realize fixed-time synchronization under controller (22). In addition,

$$
T_{\max }^{1}=\frac{2}{\alpha(1-\xi)}+\frac{2}{\beta(\eta-1)},
$$

where $\alpha=\min _{i}\left\{r_{i}\right\} 2^{(\xi+1) / 2}$ and $\beta=\min _{i}\left\{s_{i}\right\} n^{(1-\eta) / 2} \cdot 2^{(\eta+1) / 2}$.

Proof. Similarly, we can prove that

$$
\begin{aligned}
\dot{V}(e(t)) & \leq-\alpha V^{(\xi+1) / 2}(e(t))-\beta V^{(\eta+1) / 2}(e(t))-\gamma V(e(t)) \\
& \leq-\alpha V^{(\xi+1) / 2}(e(t))-\beta V^{(\eta+1) / 2}(e(t))
\end{aligned}
$$

where $\alpha=\min _{i}\left\{r_{i}\right\} 2^{(\xi+1) / 2}, \beta=\min _{i}\left\{s_{i}\right\} n^{(1-\eta) / 2} \cdot 2^{(\eta+1) / 2}$, and $\gamma=\min _{i}\left\{2 \varrho_{i}+2 k_{i}-\sum_{j=1}^{n}\left(\left|a_{i j}\right| l_{j}+\left|a_{j i}\right| l_{i}\right)\right\}>0$.

Based on Lemma 3, the origin of system (3) can achieve fixed-time stability. In addition,

$$
\begin{aligned}
T_{\max }^{1} & =\frac{1}{\alpha(1-((\xi+1) / 2))}+\frac{1}{\beta(((\eta+1) / 2)-1)} \\
& =\frac{2}{\alpha(1-\xi)}+\frac{2}{\beta(\eta-1)} .
\end{aligned}
$$


Corollary 2. Suppose assumptions $\boldsymbol{A 1}$ and $\mathbf{A 2}$ hold. If control gains $k_{i}$ and $q_{i}$ satisfy $k_{i}>\sum_{j=1}^{n}\left(\left(\left|a_{i j}\right| l_{j}+\left|a_{j i}\right| l_{i}\right) /\right.$ 2) $-\varrho_{i}, q_{i} \geq 2 \sum_{j=1}^{n}\left|b_{i j}\right| M_{j}, i=1,2, \cdots, n$, systems (1) and (2) can realize fixed-time synchronization under controller (22). In addition,

$$
T_{\max }^{2}=\frac{1}{\alpha} \cdot\left(\frac{\alpha}{\beta}\right)^{(1-\xi) /(\eta-\xi)}\left(\frac{2}{1-\xi}+\frac{2}{\eta-1}\right),
$$

where $\alpha=\min _{i}\left\{r_{i}\right\} 2^{(\xi+1) / 2}$ and $\beta=\min _{i}\left\{s_{i}\right\} n^{(1-\eta) / 2} \cdot 2^{(\eta+1) / 2}$.

Proof. Similarly, we can prove that

$$
\dot{V}(e(t)) \leq-\alpha V^{(\xi+1) / 2}(e(t))-\beta V^{(\eta+1) / 2}(e(t)),
$$

where $\alpha=\min _{i}\left\{r_{i}\right\} 2^{(\xi+1) / 2}$ and $\beta=\min _{i}\left\{s_{i}\right\} n^{(1-\eta) / 2} \cdot 2^{(\eta+1) / 2}$.

Based on Lemma 4, the origin of system (3) can achieve fixed-time stability. In addition,

$$
T_{\max }^{2}=\frac{1}{\alpha} \cdot\left(\frac{\alpha}{\beta}\right)^{(1-\xi) /(\eta-\xi)}\left(\frac{2}{1-\xi}+\frac{2}{\eta-1}\right) .
$$

Now, we consider the following controller:

$$
\begin{aligned}
u_{i}(t)= & -k_{i} e_{i}(t)-q_{i} \operatorname{sign}\left(e_{i}(t)\right)-r_{\mathrm{i}} \operatorname{sign}\left(e_{i}(t)\right)\left|e_{i}(t)\right|^{\xi} \\
& -s_{i} \operatorname{sign}\left(e_{i}(t)\right)\left|e_{i}(t)\right|^{\eta},
\end{aligned}
$$

$i=1,2, \cdots, n$, where $k_{i}$ and $q_{i}$ will be determined later, $r_{i}$ and $s_{i}$ are arbitrary positive constants, $\xi=1-(1 / \delta)$, and $\eta=1+(1 / \delta), \delta>1$.

Corollary 3. Suppose assumptions $\boldsymbol{A 1}$ and $\mathbf{A 2}$ hold. If control gains $k_{i}$ and $q_{i}$ satisfy $k_{i}>\sum_{j=1}^{n}\left(\left(\left|a_{i j}\right| l_{j}+\left|a_{j i}\right| l_{i}\right) /\right.$ 2) $-\varrho_{i}, q_{i} \geq 2 \sum_{j=1}^{n}\left|b_{i j}\right| M_{j}, i=1,2, \cdots, n$, systems (1) and (2) can realize fixed-time synchronization under controller (35). In addition,

$$
T_{\max }^{3}=\frac{\pi \delta}{\sqrt{\alpha \beta}}
$$

where $\alpha=\min _{i}\left\{r_{i}\right\} 2^{(\xi+1) / 2}$ and $\beta=\min _{i}\left\{s_{i}\right\} n^{(1-\eta) / 2} \cdot 2^{(\eta+1) / 2}$.

Proof. Similarly, we can prove that

$$
\dot{V}(e(t)) \leq-\alpha V^{(\xi+1) / 2}(e(t))-\beta V^{(\eta+1) / 2}(e(t)),
$$

where $\alpha=\min _{i}\left\{r_{i}\right\} 2^{(\xi+1) / 2}, \quad \beta=\min _{i}\left\{s_{i}\right\} n^{(1-\eta) / 2} \cdot 2^{(\eta+1) / 2}$, $((\xi+1) / 2)=1-(1 / 2 \delta),((\eta+1) / 2)=1+(1 / 2 \delta)$, and $\delta>1$.

Based on Lemma 5, the origin of system (3) can achieve fixed-time stability. In addition,

$$
T_{\max }^{3}=\frac{\pi \delta}{\sqrt{\alpha \beta}} \text {. }
$$

To study the finite-time synchronization of systems (1) and (2), we design the following controller:

$$
u_{i}(t)=-k_{i} e_{i}(t)-q_{i} \operatorname{sign}\left(e_{i}(t)\right)-r_{i} \operatorname{sign}\left(e_{i}(t)\right)\left|e_{i}(t)\right|^{\xi},
$$

$i=1,2, \cdots, n$, where $k_{i}$ and $q_{i}$ will be determined later, $r_{i}$ is an arbitrary positive constant, and $0<\xi<1$.

Corollary 4. Suppose assumptions $\boldsymbol{A 1}$ and $\mathbf{A 2}$ hold. If control gains $k_{i}$ and $q_{i}$ satisfy $k_{i}>\sum_{j=1}^{n}\left(\left(\left|a_{i j}\right| l_{j}+\left|a_{j i}\right| l_{i}\right) /\right.$ 2) $-\varrho_{i}, q_{i} \geq 2 \sum_{j=1}^{n}\left|b_{i j}\right| M_{j}, i=1,2, \cdots, n$, systems (1) and (2) can realize finite-time synchronization under controller (36). In addition,

$$
T(e(0))=\frac{1}{\alpha(1-\xi)}\left(\frac{1}{2} \sum_{i=1}^{n}\left(e_{i}(0)\right)^{2}\right)^{(1-\xi) / 2},
$$

where $\alpha=\min _{i}\left\{r_{i}\right\} 2^{(\xi+1) / 2}$.

Proof. Similarly, we can prove that

$$
\dot{V}(e(t)) \leq-\alpha V^{(\xi+1) / 2}(e(t)),
$$

where $\alpha=\min _{i}\left\{r_{i}\right\} 2^{(\xi+1) / 2}, 0<((\xi+1) / 2)<1$.

Based on Lemma 6, the origin of system (3) can achieve finite-time stability. In addition,

$$
\begin{aligned}
T(e(0)) & =\frac{V^{1-((\xi+1) / 2)}(e(0))}{\alpha(1-((\xi+1) / 2))}=\frac{2 V^{(1-\xi) / 2}(e(0))}{\alpha(1-\xi)} \\
& =\frac{1}{\alpha(1-\xi)}\left(\frac{1}{2} \sum_{i=1}^{n}\left(e_{i}(0)\right)^{2}\right)^{(1-\xi) / 2} .
\end{aligned}
$$

Remark 2. Based on the fixed-time stability theorems in $[23,24,29]$, there have been some publications about the fixed-time synchronization of neural networks [29-34]. However, the upper bound estimate formulas for the settling time given in $[23,24,29]$ are very inaccurate. In this paper, we establish a new fixed-time stability theorem, which provides a novel upper bound estimate formula for the settling time. Numerical simulations show that the upper bound estimate for the settling time in this paper is tighter than those given in $[23,24,29]$.

Remark 3. In the controllers designed in this paper, the switching item sign $\left(e_{i}(t)\right)$ is used to deal with the sign problems in theoretical derivation. In theoretical derivation, $\left|e_{i}(t)\right|$ is desirable, while $e_{i}(t)$ is not desirable. In this case, since $\operatorname{sign}\left(e_{i}(t)\right) e_{i}(t)=\left|e_{i}(t)\right|, \operatorname{sign}\left(e_{i}(t)\right)$ can play a key role. It should be pointed out that $\operatorname{sign}\left(e_{i}(t)\right)$ is a discontinuous term, which may lead to chattering phenomenon. Alternatively, the continuous term $e_{i}(t) /\left(\left|e_{i}(t)\right|+\gamma\right)$ can be used to replace $\operatorname{sign}\left(e_{i}(t)\right)$ in engineering applications, where $\gamma>0$ is a sufficiently small constant. However, in strict theoretical analysis, we need not do this kind of replacement, and this kind of replacement usually leads to problems.

\section{Numerical Simulations}

In this section, a numerical example is provided to validate the obtained theoretical results.

Example 1. Consider the following neural network: 


$$
\begin{aligned}
\dot{\xi}_{i}(t)= & -\varrho_{i} \xi_{i}(t)+\sum_{j=1}^{2} a_{i j} f_{j}\left(\xi_{j}(t)\right) \\
& +\sum_{j=1}^{2} b_{i j} f_{j}\left(\xi_{j}(t-\tau(t))\right)+I_{i},
\end{aligned}
$$

$i=1,2$, where $\varrho_{1}=0.9, \quad \varrho_{2}=1.1, \quad a_{11}=-0.5, \quad a_{12}=2$, $a_{21}=0.1, a_{22}=1.5, b_{11}=-1.2, b_{12}=1, b_{21}=0.1, b_{22}=-1.5$, $I_{1}=\sin t$, and $I_{2}=\cos t$.

Let $f_{1}(\mu)=f_{2}(\mu)=(|\mu+1|-|\mu-1|) / 2$ and $\tau(t)=1.2$; we have $M_{1}=M_{2}=1, l_{1}=l_{2}=1$, and $\tau=1.2$. The initial condition of system $(43)$ is $\varphi(\nu)=(1.5,-0.5)^{T}$, where $v \in[-1.2,0]$.

The corresponding response system is

$$
\begin{aligned}
\dot{\eta}_{i}(t)= & -\varrho_{i} \eta_{i}(t)+\sum_{j=1}^{2} a_{i j} f_{j}\left(\eta_{j}(t)\right) \\
& +\sum_{j=1}^{2} b_{i j} f_{j}\left(\eta_{j}(t-\tau(t))\right)+I_{i}+u_{i}(t),
\end{aligned}
$$

$i=1,2$, where $u_{i}(t), i=1,2$, are controllers. Suppose the initial conditions of system (44) are $\phi(\nu)=(-0.6$, $1.8)^{T},(-8,9)^{T},(-50,60)^{T}$, and $\nu \in[-1.2,0]$, respectively. The synchronization errors between systems (43) and (44) without control inputs are given in Figure 1.

Choose $k_{1}=3, k_{2}=4, q_{1}=4.4, q_{2}=3.2, r_{1}=r_{2}=1$, $s_{1}=s_{2}=1, \xi=(2 / 3)$, and $\eta=(4 / 3)$; then, we have $\alpha=$ $2^{(5 / 6)}, \beta=2, \gamma=2.7$. It can be verified that $k_{i}>\sum_{j=1}^{n}$ $\left(\left(\left|a_{i j}\right| l_{j}+\left|a_{j i}\right| l_{i}\right) / 2\right)-\varrho_{i}$ and $q_{i} \geq 2 \sum_{j=1}^{n}\left|b_{i j}\right| M_{j}, i=1,2$.

Based on Theorem 2, systems (43) and (44) can realize fixed-time synchronization under controller (22) and $T_{\max }^{4} \approx 4.6486$. The synchronization errors between systems (43) and (44) under controller (22) are presented in Figure 2. Although there are three kinds of different synchronization errors, one can see that all the synchronization errors tend to 0 within $T_{\max }^{4}$.

Remark 4. Based on Corollary 1 and Corollary 2, systems (43) and (44) can realize fixed-time synchronization under controller $(22)$, and $T_{\max }^{1} \approx 6.3674$ and $T_{\max }^{2} \approx 6.3568$. Furthermore, systems (43) and (44) can realize fixed-time synchronization under controller (35) according to Corollary $3(\delta=3)$, and $T_{\max }^{3} \approx 4.9926$. In fact, if $\xi=(2 / 3)$ and $\eta=(4 / 3)$, controller (35) is the same as controller (22). Obviously, numerical example shows that $T_{\max }^{4}$ is smaller than $T_{\max }^{1}, T_{\max }^{2}$, and $T_{\max }^{3}$ (see Figure 2).

Remark 5. Based on Corollary 4, by choosing the same parameters as those in Example 1, systems (43) and (44) can realize finite-time synchronization under controller (36). It should be pointed out that the settling time $T(e(0))$ is related to $e(0)$, which depends on the initial values of systems (43) and (44). Therefore, the settling time $T(e(0))$ cannot be bounded by a unified upper bound. If $\|e(0)\|$ is large enough, the settling time $T(e(0))$ will be larger than $T_{\max }^{4}$. In contrast, for arbitrary $e(0)$, systems (43) and (44) can realize

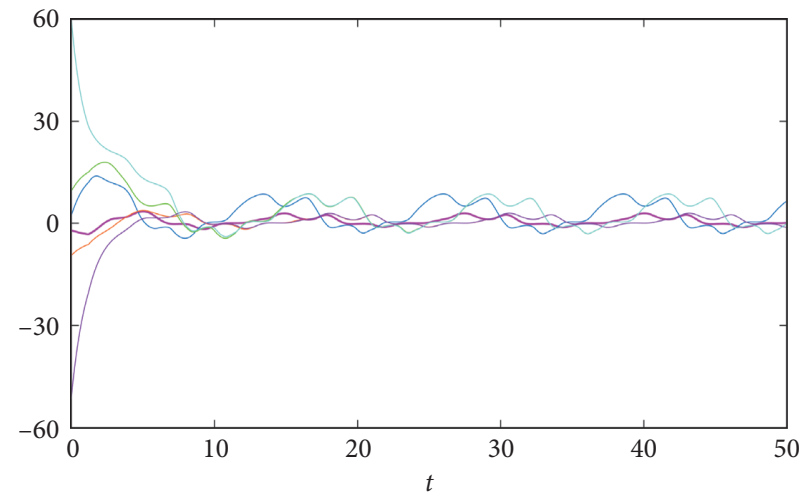

Figure 1: The synchronization errors between systems (43) and (44) without control inputs, where the initial condition of system (43) is $\varphi(\nu)=(1.5,-0.5)^{T}$ and the initial conditions of system (44) are $\phi(\nu)=(-0.6,1.8)^{T},(-8,9)^{T},(-50,60)^{T}$, and $\nu \in[-1.2,0]$, respectively.

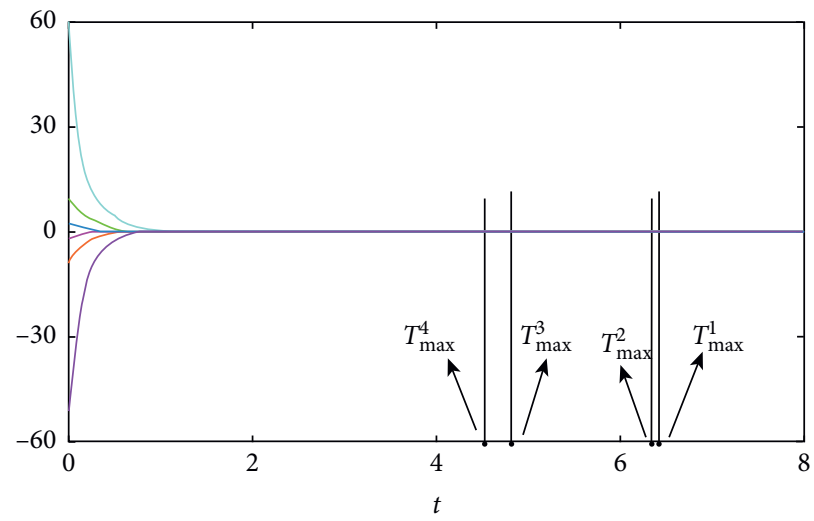

Figure 2: The synchronization errors between system (22) under controller (22), where the initial condition of system (43) is $\varphi(\nu)=(1.5,-0.5)^{T}$, and the initial conditions of system $(44)$ are $\phi(\nu)=(-0.6,1.8)^{T},(-8,9)^{T},(-50,60)^{T}$, and $\nu \in[-1.2,0]$, respectively.

fixed-time synchronization under controller (22) within $T_{\max }^{4}$.

Remark 6. In Example 1, if we choose some larger $k_{i}$ and keep the other controller parameters unchanged, the obtained $T_{\max }^{4}$ can be smaller. However, $T_{\max }^{1}, T_{\max }^{2}$, and $T_{\max }^{3}$ still remain unchanged in this case.

\section{Conclusions}

In this paper, the fixed-time synchronization of neural networks with discrete delay is investigated by utilizing the newly developed fixed-time stability theorem, which can give the settling time a tighter upper bound estimate compared with the existing fixed-time stability theorems. The settling time of fixed-time synchronization/stability is bounded by a fixed constant, irrespective of the initial conditions of the considered systems. The obtained fixedtime synchronization criteria can be verified easily, and 
numerical simulations are provided to demonstrate the validity of the theoretical results. In the future, we will study the application of neural networks in associative memory.

\section{Data Availability}

The data used to support the findings of this study are available from the corresponding author upon request.

\section{Disclosure}

Julian Shen and Wei Wei are co-first authors.

\section{Conflicts of Interest}

The authors declare that they have no conflicts of interest.

\section{Acknowledgments}

This work was supported by the National Natural Science Foundation of China (Grant nos. 61771071 and 11771196).

\section{References}

[1] J. J. Hopfield, "Neurons with graded response have collective computational properties like those of two-state neurons," Proceedings of the National Academy of Sciences, vol. 81, no. 10, pp. 3088-3092, 1984.

[2] K. Aihara, T. Takabe, and M. Toyoda, "Chaotic neural networks," Physics Letters A, vol. 144, no. 6-7, pp. 333-340, 1990.

[3] G. Bao and Z. Zeng, "Analysis and design of associative memories based on recurrent neural network with discontinuous activation functions," Neurocomputing, vol. 77, no. 1, pp. 101-107, 2012.

[4] J. Zhou, T. Chen, and L. Xiang, "Chaotic lag synchronization of coupled delayed neural networks and its applications in secure communication," Circuits, Systems \& Signal Processing, vol. 24, no. 5, pp. 599-613, 2005.

[5] S. Wen, Z. Zeng, T. Huang, Q. Meng, and W. Yao, "Lag synchronization of switched neural networks via neural activation function and applications in image encryption," IEEE Transactions on Neural Networks and Learning Systems, vol. 26, no. 7, pp. 1493-1502, 2015.

[6] B. Yang, R. Wang, and G. M. Dimirovski, "Delay-dependent stability for neural networks with time-varying delays via a novel partitioning method," Neurocomputing, vol. 173, pp. 1017-1027, 2016.

[7] L. Song, S. K. Nguang, and D. Huang, "Hierarchical stability conditions for a class of generalized neural networks with multiple discrete and distributed delays," IEEE Transactions on Neural Networks and Learning Systems, vol. 30, no. 2, pp. 636-642, 2019.

[8] X. Yang, Q. Song, J. Cao, and J. Lu, "Synchronization of coupled markovian reaction-diffusion neural networks with proportional delays via quantized control," IEEE Transactions on Neural Networks and Learning Systems, vol. 30, no. 3, pp. 951-958, 2019.

[9] A. Pratap, R. Raja, J. Cao, C. P. Lim, and O. Bagdasar, "Stability and pinning synchronization analysis of fractional order delayed Cohen-Grossberg neural networks with discontinuous activations," Applied Mathematics and Computation, vol. 359, pp. 241-260, 2019.
[10] J. Hou, Y. Huang, and S. Ren, "Anti-synchronization analysis and pinning control of multi-weighted coupled neural networks with and without reaction-diffusion terms," Neurocomputing, vol. 330, pp. 78-93, 2019.

[11] Z. Cai and L. Huang, "Finite-time stabilization of delayed memristive neural networks: discontinuous state-feedback and adaptive control approach," IEEE Transactions on Neural Networks and Learning Systems, vol. 29, no. 4, pp. 856-868, 2018.

[12] C. Chen, L. Li, H. Peng, and Y. Yang, "Adaptive synchronization of memristor-based BAM neural networks with mixed delays," Applied Mathematics and Computation, vol. 322, pp. 100-110, 2018.

[13] J. Mei, M. Jiang, X. Wang, J. Han, and S. Wang, "Finite-time synchronization of drive-response systems via periodically intermittent adaptive control," Journal of the Franklin Institute, vol. 351, no. 5, pp. 2691-2710, 2014.

[14] X. Yang, Q. Song, J. Liang, and B. He, "Finite-time synchronization of coupled discontinuous neural networks with mixed delays and nonidentical perturbations," Journal of the Franklin Institute, vol. 352, no. 10, pp. 4382-4406, 2015.

[15] J. Shen and J. Cao, "Finite-time synchronization of coupled neural networks via discontinuous controllers," Cognitive Neurodynamics, vol. 5, no. 4, pp. 373-385, 2011.

[16] H. Shen, J. H. Park, and Z.-G. Wu, "Finite-time synchronization control for uncertain Markov jump neural networks with input constraints," Nonlinear Dynamics, vol. 77, no. 4, pp. 1709-1720, 2014.

[17] J. Huang, C. Li, T. Huang, and X. He, "Finite-time lag synchronization of delayed neural networks," Neurocomputing, vol. 139, pp. 145-149, 2014.

[18] C. Chen, L. Li, H. Peng, Y. Yang, and T. Li, "Finite-time synchronization of memristor-based neural networks with mixed delays," Neurocomputing, vol. 235, pp. 83-89, 2017.

[19] C. Hu, J. Yu, and H. Jiang, "Finite-time synchronization of delayed neural networks with Cohen-Grossberg type based on delayed feedback control," Neurocomputing, vol. 143, pp. 90-96, 2014.

[20] X. Liu, J. Cao, W. Yu, and Q. Song, "Nonsmooth finite-time synchronization of switched coupled neural networks," IEEE Transactions on Cybernetics, vol. 46, no. 10, pp. 2360-2371, 2016.

[21] M. Zheng, L. Li, H. Peng, J. Xiao, Y. Yang, and H. Zhao, "Finite-time projective synchronization of memristor-based delay fractional-order neural networks," Nonlinear Dynamics, vol. 89, no. 4, pp. 2641-2655, 2017.

[22] D. Huang, M. Jiang, and J. Jian, "Finite-time synchronization of inertial memristive neural networks with time-varying delays via sampled-date control," Neurocomputing, vol. 266, pp. 527-539, 2017.

[23] A. Polyakov, "Nonlinear feedback design for fixed-time stabilization of linear control systems," IEEE Transactions on Automatic Control, vol. 57, no. 8, pp. 2106-2110, 2012.

[24] S. Parsegov, A. Polyakov, and P. Shcherbakov, "Nonlinear fixed-time control protocol for uniform allocation of agents on a segment," in Proceedings of the IEEE 51st Annual Conference on Decision and Control, pp. 7732-7737, Maui, HI, USA, December 2012.

[25] J. Ni, L. Liu, C. Liu, X. Hu, and S. Li, "Fast fixed-time nonsingular terminal sliding mode control and its application to chaos suppression in power system," IEEE Transactions on Circuits and Systems II: Express Briefs, vol. 64, no. 2, pp. 151-155, 2017. 
[26] X. Liu and T. Chen, "Finite-time and fixed-time cluster synchronization with or without pinning control," IEEE Transactions on Cybernetics, vol. 48, no. 1, pp. 240-252, 2018.

[27] C. Chen, L. Li, H. Peng, J. Kurths, and Y. Yang, "Fixed-time synchronization of hybrid coupled networks with timevarying delays," Chaos, Solitons \& Fractals, vol. 108, pp. 4956, 2018.

[28] G. Ji, C. Hu, J. Yu, and H. Jiang, "Finite-time and fixed-time synchronization of discontinuous complex networks: a unified control framework design," Journal of the Franklin Institute, vol. 355, no. 11, pp. 4665-4685, 2018.

[29] C. $\mathrm{Hu}, \mathrm{J} . \mathrm{Yu}, \mathrm{Z}$. Chen, H. Jiang, and T. Huang, "Fixed-time stability of dynamical systems and fixed-time synchronization of coupled discontinuous neural networks," Neural Networks, vol. 89, pp. 74-83, 2017.

[30] Y. Wan, J. Cao, G. Wen, and W. Yu, "Robust fixed-time synchronization of delayed Cohen-Grossberg neural networks," Neural Networks, vol. 73, pp. 86-94, 2016.

[31] X. Ding, J. Cao, A. Alsaedi, F. E. Alsaadi, and T. Hayat, "Robust fixed-time synchronization for uncertain complexvalued neural networks with discontinuous activation functions," Neural Networks, vol. 90, pp. 42-55, 2017.

[32] Y. Xu, D. Meng, C. Xie, G. You, and W. Zhou, "A class of fast fixed-time synchronization control for the delayed neural network," Journal of the Franklin Institute, vol. 355, no. 1, pp. 164-176, 2018.

[33] J. Cao and R. Li, "Fixed-time synchronization of delayed memristor-based recurrent neural networks," Science China Information Sciences, vol. 60, no. 3, pp. 1-15, Article ID 032201, 2017.

[34] C. Chen, L. Li, H. Peng, and Y. Yang, "Fixed-time synchronization of memristor-based BAM neural networks with time-varying discrete delay," Neural Networks, vol. 96, pp. 47-54, 2017.

[35] G. Hardy, J. Littlewood, and G. Polya, Inequalities, Cambridge University Press, Cambridge, UK, 1952.

[36] Y. Tang, "Terminal sliding mode control for rigid robots," Automatica, vol. 34, no. 1, pp. 51-56, 1998. 